\title{
Efecto lactogénico de la adición de harina de fenogreco (Trigonella foenum-graecum L.) sobre el rendimiento de cabras en período de lactación
}

\author{
Kevin Donaldo Véliz García ${ }^{1}$ - Carlos Enrique Corzantes Cruz² - Déborah Cecilia Rodríguez Sánchez ${ }^{3}$
}

Recepción: 26/05/2019 Aceptación: 06/08/2019

\section{Resumen}

El presente estudio se realizó con el fin de evaluar el impacto lactogénico al agregar harina de fenogreco a la dieta de cabras lactantes. Se dividió en dos grupos a doce cabras lactantes, teniendo en cuenta la condición corporal, el número de lactancia, la edad y la etapa de lactación de cada una de ellas. El primer grupo se tomó como control y no recibió tratamiento; al segundo grupo, se le agregó a la dieta 40 gramos de harina de fenogreco diarios.

El ordeño se realizó dos veces al día, y se ofrecían 20 gramos antes de cada uno. El resultado del análisis de la producción demostró que el rendimiento de leche fue significativamente mayor $(\mathrm{P}<0.05)$ en las cabras tratadas con fenogreco en comparación con el grupo control.

Al finalizar el estudio se tomaron dos muestras de la leche obtenida, con la finalidad de evaluar la composición de esta en las dos muestras evaluadas. También se realizó un análisis sensorial con estudiantes de la Facultad de Medicina Veterinaria y Zootecnia de cuarto ciclo para determinar diferencias en el sabor, olor y textura de la leche. Se puede concluir que la alimentación con el fenogreco incrementó la producción de leche.

Palabras clave: Galactogogos naturales, producción láctea, harina de fenogreco, cabras.

\begin{abstract}
This study was conducted to evaluate the lactogenic impact of adding fenugreek flour to the diet of lactating goats. Twelve lactating goats were divided into two groups, taking into account the body condition, lactation number, and age and lactation stage of each goat. The first group was taken as a control and received no treatment; to the second group, 40 grams of fenugreek flour were added to the diet daily.
\end{abstract}

Milking took place twice a day, and 20 grams were offered before each. Then result of the production analysis showed that milk yield was significantly higher (P 0.05) in fenugreek-treated goats compared to the control group.

At the end of the study, two samples were taken from the milk obtained in order to evaluate the composition of the milk in the two samples evaluated. A sensory analysis was also conducted with students from the faculty Veterinary Medicine and Fourth Cycle Animal Husbandry to determine differences in milk taste, smell and texture. It can be concluded that feeding fenugreek increased milk production.

Key words: Natural galactagogic, dairy production, fenugreek flour, goats.

\footnotetext{
1. Pasante de la licenciatura en Zootecnia, Universidad de San Carlos de Guatemala - Guatemala; email: kevin.velizg.89@gmail.com

2. Catedrático nivel básico, Universidad de San Carlos de Guatemala - Guatemala; email: cenriquecc@gmail.com

3. Catedrática nivel introductorio, Universidad de San Carlos de Guatemala - Guatemala; email: debrodris@gmail.com
} 


\section{Introducción}

El deseo que se presenta en la mayoría de productores es aumentar el rendimiento del rebaño, para ello se ha desarrollado una amplia variedad de métodos que han incluido mejoras en la genética y en las raciones alimenticias. Recientemente se ha utilizado el mestizaje de las cabras para mejorar la producción y composición de la leche, pero hace variar los recursos genéticos importantes. Además, este mestizaje puede modificar alguna de las características que los productores valoran en sus animales, ya que genéticamente existe la dilución de la raza que hace que, según (Dickson et al., 2001), los animales disminuyan o alteren la producción; también se han desarrollado aditivos que pueden ayudar en obtener una alta producción.

Entre los aditivos que se han desarrollado, según menciona Acedo-Rico (2003), se pueden encontrar enzimas, levaduras y minerales de forma orgánica que incrementan la producción de leche pero alteran sus propiedades, haciendo que pierda palatabilidad. El efecto de estos aditivos hace que se favorezca la digestibilidad de celulosa en los forrajes, es por esto que resulta necesario incorporar alternativas naturales seguras y aplicables con el fin de mejorar la producción láctea caprina.

El fenogreco (Trigonella foenum-graecum L.) contiene galactogogos naturales que estimulan la producción láctea si se administra en una mezcla adecuada durante unos días en época de lactancia. Según Snehlata y Payal (2012), después de su carácter inmunológico, las semillas de fenogreco contienen precursores de hormonas que aumentan la producción de leche. Algunos científicos creen que es posible debido a que las glándulas mamarias son sudoríparas modificadas, y el fenogreco estimula la producción de sudor. Esto mismos autores han demostrado que el fenogreco puede aumentar la producción de leche de una madre lactante en un período de entre 24 a 72 horas después de administrarlo (Snehlata \& Payal, 2012).

El fenogreco es una de las plantas que se ha usado a través de la historia para mejorar el suministro de leche. Algunas plantas mencionadas como galactogogos incluyen fenogreco (Trigonella foenum-graecum L), anís (Pimpinella anisum), semillas de hinojo (Foeniculum vulgare), ixbut (Euphoria lancifolia) (Cáceres, 2014). Se desconocen cuáles son exactamente los mecanismos de acción de la mayoría de las plantas galactagogas, debido a que la mayoría no han sido evaluadas científicamente, pero su uso tradicional sugiere su seguridad y eficacia (Laborales y Con, 2014).

El fenogreco es una planta que pertenece a la familia de las Fabáceas, subfamilia Faboideae, esta planta puede encontrarse en diversas partes del sur de Europa, pero se cultiva principalmente en el Medio Oriente. La semilla de 
esta planta es aromática, amarga carminativa y galactagoga. Este estudio fue diseñado para evaluar el efecto que tiene la harina del fenogreco en la ración alimenticia diaria de cabras lactantes en condiciones del trópico.

\section{Materiales y Métodos}

Se trabajó con doce cabras lactantes sin raza definida (SRD) de 2-3 años, de similar condición corporal y producción láctea; todas ellas en segunda lactancia. Las cabras fueron asignadas aleatoriamente a dos grupos de seis unidades experimentales cada uno: el grupo testigo y el grupo experimental. Ambos grupos fueron nutridos con tres libras de alimento balanceado, dos horas con pasto Swazi (Digitaria swazilandensis), después del ordeño en parcelas exclusivas para el pastoreo. Al grupo experimental, durante cinco semanas se le adicionó a la dieta, 40 gramos de harina de fenogreco. Luego de un análisis de laboratorio, se determinó que el fenogreco tiene en su composición $13.68 \%$ de proteína cruda (PC), 63.67\% extracto libre de nitrógeno (ELN); $16.56 \%$ Fibra Cruda (FC) y $2.63 \%$ Extracto etéreo $(\mathrm{EE})$.

Esta harina de fenogreco se ofertó desde el inicio sin hacer ningún período de adaptación a la dieta, ya que se deseaba conocer el período en el que el fenogreco tenía un efecto. Esta harina fue adquirida en laboratorios y droguería "Quinfica” que se encarga de la venta de medicamentos, fitoterapéuticos $y$ cosméticos en Guatemala.
En la finca se efectúan dos ordeños manuales. Diariamente se recolectó la producción y se tomaron los registros a las 7:00 y 18:00, individualmente, durante las cinco semanas que duró el estudio.

\begin{tabular}{|c|c|}
\hline Control (2) & Tratamiento(1) \\
\hline $\begin{array}{c}\text { Alimento } \\
\text { formulado }\end{array}$ & $\begin{array}{c}\text { Alimento formulado y } 40 \\
\text { gramos de fenogreco }\end{array}$ \\
\hline
\end{tabular}

Figura 1. Dietas diarias ofertadas durante el estudio.

Este estudio se llevó a cabo en la aldea Cuyuta, perteneciente al municipio de Masagua del departamento de Escuintla, Guatemala, que se encuentra a 15 m.s.n.m., en donde predomina el clima tropical.

Tabla 1. Alimento ofertado durante el estudio.

\begin{tabular}{|c|c|}
\hline Ingrediente & $\begin{array}{c}\text { Porcentaje de } \\
\text { inclusión }\end{array}$ \\
\hline Maíz molido & $65 \%$ \\
\hline Harina de soya & $20 \%$ \\
\hline Melaza & $4.5 \%$ \\
\hline Grasa & $4.7 \%$ \\
\hline Bicarbonato de calcio & $2 \%$ \\
\hline Sal Blanca & $1 \%$ \\
\hline Sales minerales & $1.5 \%$ \\
\hline Urea & $1.3 \%$ \\
\hline
\end{tabular}

\section{Resultados y Discusión}

El efecto del fenogreco se pudo observar en la segunda semana cuando el promedio del grupo suplementado con harina de fenogreco alcanzó 9.1 litros, a diferencia del grupo control, que en la misma fecha alcanzó 6.88 litros. 
Los dos grupos iniciaron con similar promedio: 8.33 litros para el grupo que sería suplementado con la harina de fenogreco (1) y 7.66 litros para el grupo control (2). Conforme fue transcurriendo el tiempo, el grupo con tratamiento fue aumentando la producción progresivamente (ver tablas 2 y 3 ).

Tabla 2. Efecto lactogénico de la harina de fenogreco ( 1 por semana) en cabras lactantes

\begin{tabular}{|c|c|c|}
\hline \multirow{2}{*}{$\begin{array}{c}\text { Semana de } \\
\text { tratamiento }\end{array}$} & \multicolumn{2}{|c|}{ Grupos } \\
\cline { 2 - 3 } & $\begin{array}{c}\text { Con } \\
\text { tratamiento } \\
(\mathbf{1})\end{array}$ & $\begin{array}{c}\text { Sin } \\
\text { tratamiento } \\
\mathbf{( 2 )}\end{array}$ \\
\hline Primera & $8.33 \mathrm{~L}$ & $7.67 \mathrm{~L}$ \\
\hline Segunda & $9.1 \mathrm{~L}$ & $6.88 \mathrm{~L}$ \\
\hline Tercera & $8.88 \mathrm{~L}$ & $6.68 \mathrm{~L}$ \\
\hline Cuarta & $8.37 \mathrm{~L}$ & $5.17 \mathrm{~L}$ \\
\hline Quinta & $4.05 \mathrm{~L}$ & $3.28 \mathrm{~L}$ \\
\hline
\end{tabular}

El análisis de varianza únicamente encontró diferencias significativas entre tratamientos y los distintos períodos (semanas), por lo que se precedió a realizar la prueba de medias de Tukey para esas variables clasificatorias (ver tablas 6 y 7 ).

El cuadro de Análisis de Varianza encontró diferencia significativa entre tratamientos $(\mathrm{p}<0.05)$, mostrando la prueba de medias que el tratamiento 1 (Alimento formulado y 40 gramos de harina de fenogreco) fue con el que las cabras produjeron mayor cantidad de leche $(0.58 \mathrm{~L})$ en promedio; mientras que con el tratamiento 2 (Alimento formulado), produjeron 0.44 litros.
También el ANDEVA determinó que entre períodos (semanas) se encontró diferencias significativas $(\mathrm{p}<0.05)$, indicando la prueba de medias (Tukey) que los períodos 1, 2, 3, no hubo diferencias en cuanto a la producción de leche $(0.57,0.57$ y 0.56 litros respectivamente); mientras que entre los períodos 4 y 5, tampoco se encontraron diferencias significativas, obteniendo producciones de 0.47 y 0.37 , correspondientemente. En resumen, la prueba de medias detectó dos grupos entre los cuales hubo diferencia estadística significativa: el primero conformado por los períodos 1, 2 y 3; y el segundo grupo, por los períodos 4 y 5 .

Los resultados obtenidos en el presente estudio son similares a los obtenidos por Khaliq (2012) y El-Tarabany et al. (2017) en los que se encontró un aumento significativo en la producción de leche, mas no así en otros componentes que la conforman. El aumento de la producción puede ser por medio de la hormona prolactina que presenta un posible mecanismo endocrino en la acción del fenogreco. Esto no está definido, debido a que Smit (2014), aseguró que la producción en animales se puede mejorar con plantas medicinales, y que los efectos hormonales como la prolactina y la hormona de crecimiento circulan regularmente en el cuerpo. Los tejidos de la ubre se activan con el aumento de la glucosa y con una disminución en la concentración de colesterol en la sangre. 
Tabla 3. Promedio obtenido por animal semanalmente

\begin{tabular}{|c|c|c|c|c|c|c|}
\hline Tratamiento & Animal & Periodo 1 & Periodo 2 & Periodo 3 & Periodo 4 & Periodo 5 \\
\hline 1 & 1 & 0.493 & 0.629 & 0.693 & 0.671 & 0.45 \\
\hline 1 & 2 & 0.814 & 0.864 & 0.657 & 0.671 & 0.47 \\
\hline 1 & 3 & 0.736 & 0.736 & 0.643 & 0.65 & 0.43 \\
\hline 1 & 4 & 0.693 & 0.721 & 0.6 & 0.521 & 0.33 \\
\hline 1 & 5 & 0.393 & 0.471 & 0.564 & 0.564 & 0.38 \\
\hline 1 & 6 & 0.443 & 0.479 & 0.65 & 0.507 & 0.37 \\
\hline 2 & 1 & 0.329 & 0.343 & 0.507 & 0.357 & 0.19 \\
\hline 2 & 2 & 0.679 & 0.536 & 0.471 & 0.443 & 0.4 \\
\hline 2 & 3 & 0.6 & 0.6 & 0.457 & 0.436 & 0.36 \\
\hline 2 & 4 & 0.586 & 0.479 & 0.514 & 0.321 & 0.29 \\
\hline 2 & 5 & 0.471 & 0.436 & 0.414 & 0.321 & 0.32 \\
\hline 2 & 6 & 0.621 & 0.557 & 0.5 & 0.136 & 0.41 \\
\hline
\end{tabular}

\section{Análisis de Varianza en Infostat}

Tabla 4. Análisis de la varianza

\begin{tabular}{|c|c|c|c|c|}
\hline Variable & $\mathbf{N}$ & $\mathbf{R}^{\mathbf{2}}$ & $\mathbf{R}^{\mathbf{2}} \mathbf{A j}$ & $\mathbf{C V}$ \\
\hline Proleche & 60 & 0.58 & 0.50 & 20.75 \\
\hline
\end{tabular}

Tabla 5. Análisis de varianza (SC tipo III)

\begin{tabular}{|c|c|c|c|c|c|}
\hline F. V. & SC & gal & CM & F & P -valor \\
\hline Modelo & 0.76 & 9 & 0.08 & 7.68 & $<0.0001$ \\
\hline Tratamiento & 0.30 & 1 & 0.30 & 26.74 & $<0.0001$ \\
\hline Periodo & 0.38 & 4 & 0.10 & 8.68 & $<0.0001$ \\
\hline Tratamiento ${ }^{\text {Periodo }}$ & 0.08 & 4 & 0.02 & 1.90 & 0.1251 \\
\hline Error & 0.55 & 50 & 0.01 & & \\
\hline Total & 1.31 & 59 & & & \\
\hline
\end{tabular}

Tabla 6. Test: Tukey Alfa $=0.05 \mathrm{DMS}=0.05449$

\begin{tabular}{|c|c|c|c|c|}
\hline Tratamiento Medias & Medias & n & E. E. & \\
\hline $\mathbf{1}$ & 0.58 & 30 & 0.02 & A \\
\hline $\mathbf{2}$ & 0.44 & 30 & 0.02 & B \\
\hline
\end{tabular}

Nota: Medias con una letra común no son significativamente diferentes ( $\mathrm{p}>0.05)$. 
Tabla 7. Test: Tukey Alfa=0.05 DMS=0.12139

\begin{tabular}{|c|c|c|c|c|}
\hline Periodo & Medias & n & E. E. & \\
\hline $\mathbf{1}$ & 0.57 & 12 & 0.03 & $\mathrm{~A}$ \\
\hline $\mathbf{2}$ & 0.57 & 12 & 0.03 & $\mathrm{~A}$ \\
\hline $\mathbf{3}$ & 0.56 & 12 & 0.03 & $\mathrm{~A}$ \\
\hline $\mathbf{4}$ & 0.47 & 12 & 0.03 & $\mathrm{~B}$ \\
\hline $\mathbf{5}$ & 0.37 & 12 & 0.03 & $\mathrm{~B}$ \\
\hline
\end{tabular}

Nota: Medias con una letra común no son significativamente diferentes ( $p>0.059$.

Error: 0.0110 gal: 50

El-Tarabany, Teama y Atta (2017) en sus estudios determinaron que, el aumento de la producción puede ser debido al componente estrogénico que contiene el fenogreco. Esta leche fue analizada y también se observó una disminución de grasa significativa, (Khaliq, 2012). Además, tuvo resultados que demostraron que el rendimiento de un grupo evaluado fue mayor a la segunda semana, pero no hubo una disminución significativa en el porcentaje de grasa en la leche de los grupos 1 y 2 en comparación con el grupo control.

Alkas y Kawa (2010) concluyeron que las razas negras de Meriz habían tenido una diferencia significativa en la producción. La discrepancia pudo surgir por la genética, el manejo y la alimentación, debido a que la raza negra de Meriz posee el tamaño mayor entre las dos y; por consiguiente, una ingesta mayor. Concluyó en su estudio que si existe diferencia en la producción entre razas, y esto da la posibilidad a mejorar ese rasgo genético que la raza posee.
El mecanismo por el cual funciona el fenogreco no se conoce. El-Tarabany et al. (2017) menciona que a pesar que se han realizado varios estudios, no se conoce el efecto lactogénico. Esto puede ser mediado debido a que el fenogreco contiene un químico que tiene una función similar al estrógeno. Balgees et al. (2013) en sus conclusiones recomiendan que se utilicen las dietas con plantas medicinales, que mejoran el rendimiento productivo en la lactación en la cabra.

Del Villar (2010) sugiere que esto se puede utilizar, pero se debe tener un máximo cuidado al momento de suplementarlo, debido a que el fenogreco puede tener efectos oxitócicos. Estudios han demostrado que el fenogreco ha tenido un efecto estimulante en el útero, por lo cual no es recomendable durante la gestación, específicamente en dosis mayores dentro de la alimentación. 


\section{Conclusiones}

Según los resultados obtenidos, la producción fue mayor en la dieta adicionada con harina de fenogreco, demostrando que el efecto lactogénico tuvo su efecto. Se mostró un aumento en la producción de leche total en un $30.49 \%$

El efecto lactogénico se mostró a la segunda semana. Al comienzo del estudio, ambos grupos iniciaron con promedio de producción similar: 8.33 litros para el grupo suplementado con harina de fenogreco; y 7.66 litros para el grupo en el que no se utilizó. Para la segunda semana se alcanzaron 9.1 litros para el grupo 1 y 6.88 litros para el grupo 2, por lo que se puede concluir que el efecto de la harina tarda un promedio de 12 a 15 días para que haga su debido efecto.

Cabe añadir que, se recomienda realizar una prueba físico-química a las muestras de leche para conocer el efecto que pudiera existir al suplementar a las cabras con harina de fenogreco, así como también una prueba organoléptica para conocer si existe un cambio en el aroma, el gusto y la condición.

Finalmente, esta investigación no hubiera sido posible sin la colaboración de todas las personas involucradas dentro del proceso de: planificación, gestión y desarrollo. Así como de todos aquellos que apoyaron anímica y moralmente a los involucrados con orientación y escucha.

\section{Referencias}

Alkas, J. y Kawa, M. (2011). Milk production traits of indigenous Black and Meriz goats raised under farm production system. Research opinions in animal and veterinary sciences, 1 (11), 708-713. Recuperado de https://www.researchgate.net/publication/272421657_ Milk_production_traits_of_indigenous_Black_and_Meriz_goats_raised_under_farm_ production_system

Balgees A., A. E..; Siham, R. y Abdelnasir, F. (septiembre de 2013). Effect of Fenugreek Seeds Supplementation on Nutritional Performance and Milk Production of Sudanese Nubian Goats. Agricultural development within the rural-urban continuum. Congreso llevado a cabo en Tropentag, Stuttgart-Hohenheim, Alemania. Recuperado de http://www.tropentag.de/2013/abstracts/posters/622.pdf

Castillo C., C. M. (2014). Conocimiento y práctica en el uso del ixbut (Tesis de pregrado). Universidad Rafael Landívar, Fraijanes, Guatemala. Recuperado de http://biblio3.url.edu.gt/ Tesario/2014/09/03/Castillo-Claudia.pdf 
Del Villar, A. (2010). Guía de plantas medicinales del Magreb. Recuperado de http://www.areasaludbadajoz.com/images/datos/elibros/guia_plantas_medicinales_magreb.pdf

El-Tarabany, A. A.; Teama, F. E. I. y Atta, M. A. A. (2017). Effect of Fenugreek Supplementation on Physiological Functions and Milk Traits of Heat Stressed Lactating Baladi Goats. Arab Journal of Nuclear Science and Applications, 50(2), 218-228. Recuperado de https://pdfs.semanticscholar.org/f408/40fd9101267e4046e08bf902f338cf4a4e81.pdf?_ $\mathrm{ga}=2.74614161 .242937083 .1556761170-717645719.1556761170$

Helambe S., S. y Dande R., P. (2012). Fenugreek (Trigonella foenum-graecum L.): An Overview. International Journal of Current Pharmaceutical Review and Research, 2(4), 906-924. Recuperado de https://pdfs.semanticscholar.org/b784/c1b0c3582a5468fbaf866bbd0b8c8bbe9219.pdf

Khaliq, A. (2012). Feeding effects of fenugreek seeds (Tringonella foenum- graceum) on lactation performance, some serum constituents and prolactin hormone level in damascus crossbred goats Abdul. Diyala Agricultural Sciences Journal, 4 (1), 1-8. Recuperado de https://agriculmag.uodiyala.edu.iq/uploads/pdf/adad\%20 1\%202012/\%D 8\%AF. \%D 8\%B9\%D8\%A 8\%D8\%AF\%20\%D 8\%A7\%D9\% 84\%D8\% AE \% D 8\%A 7\% D 9\% 84\%D 9\% 82\% 20\% D 8\%A 7\% D 8\%AD\%D 9\% 85\% D 8\%AF\% 20 \%D9\%81\%D8\%B1\%D8\%AD\%D8\%A7\%D9\%86\%20\%D8\%A7\%D9\%84\%D8\%AC\%D9\%8 6\%D8\%A7\%D8\%A8\%D9\%8A.pdf

Portillo E., E. G. (2009). Estudio de la variabilidad genetica del piñon (Jatropha curcas L.) y actividades relacionadas con el cultivo en las comunidades de Llanitos y Las Guacas, Masagua, Escuintla (Trabajo de grado). Universidad de San Carlos de Guatemala, Guatemala. Recuperado de http://www.repositorio.usac.edu.gt/8964/1/Edan\%20Giovanni\%20Portillo\%20Eguizabal.pdf

Smit J., H. P. (2014). The effect of a natural feed additive, fenugreek, on feed digestibility and milk response in dairy goats. (Tesis de maestría). Stellenbosch University, El Cabo Occidental, Sudáfrica. Recuperado de https://scinapse.io/papers/2116791336

Vásquez M., M.; Romero C., Á. y Rivas C., A. (2014). Prácticas colombianas galactogogas y tradiciones para evitar complicaciones mamarias: papel de enfermería. Revista Ciencia y Salud Virtual, 6(1), 14-24. https://doi.org/10.22519/21455333.411 